comparing both provider and contract referral separately against patient referral.

Methods We recruited 10 health advisers from contrasting settings to attend a 1-day workshop led by partner notification specialists and researchers. They participated in focus group discussions, observations of practice, role play with each other and with actors. They and the actors then contributed to further focus groups reflecting on their observations and experiences, and advising on feasible strategies for delivering standard partner notification interventions in the RCT context. All discussions were recorded for qualitative analysis.

Results All health advisers practised provider referral, but the extent was variable. Contract referral as defined in the UK (a time period is explicitly agreed for the patient to notify, after which provider notification is initiated) is practised for HIV, other bloodborne viruses and sometimes syphilis. However for common bacterial STIs, a patient's choice of provider referral often emerges over multiple consultations, and provider referral is not a distinct intervention from contract referral. Health advisers saw their role as helping patients find solutions to partner notification, rather than applying specific interventions.

Conclusions Provider and contract referral are not sufficiently distinct to be compared with each other in a trial setting for chlamydia and gonorrhoea. However contract referral does have an important role in management of partner notification for bloodborne viruses.

\section{P5-S5.05 PARTNER NOTIFICATION UPTAKE FOR SEXUALLY TRANSMITTED INFECTIONS IN CHINA: A SYSTEMATIC REVIEW}

doi:10.1136/sextrans-2011-050108.554

${ }^{1} \mathrm{~A}$ Wang, ${ }^{2} \mathrm{R}$ Peng, ${ }^{3} \mathrm{~J}$ Tucker, ${ }^{2} \mathrm{X} S \mathrm{~S}$ Chen, ${ }^{4} \mathrm{M}$ Cohen. ${ }^{1}$ The University of Texas Medical School at Houston, Houston, USA; ${ }^{2}$ National Center for STD Control, Chinese Academy of Medical Sciences and Peking Union Medical College, Institute of Dermatology, China; ${ }^{3}$ Harvard University, USA; ${ }^{4}$ University of North Carolina, School of Medicine, USA

Background China's sexually transmitted HIV and resurgent syphilis epidemics require comprehensive control programs. Partner services are traditional pillars of sexually transmitted infection (STI) control but have not been widely implemented in China. This systematic review of English and Chinese literature examined the feasibility and uptake of partner notification (PN) in China.

Methods Four English language and four Chinese language databases were searched from 1980 to 2010 to identify original articles on partner services in China. A systematic review was performed to assess: (1) willingness to notify via patient, provider, or contract notification; and (2) PN uptake, defined as the number of partners successfully notified, evaluated, and treated per index case. Approaches to partner services in China, perceived barriers, and implementation consequences were also abstracted. $\chi^{2}$ tests were used to compare the proportions of index patients willing to notify partners using different PN methods.

Results A total of 441 citations were identified, and 17 studies (3 English and 14 Chinese), published between 1997 and 2010, examined PN willingness and uptake. All three types of PN-patient, provider, and contract-were assessed. Overall willingness to notify was $67 \%(S D \pm 25.0)$ in nine studies $(n=2145)$. Six studies $(n=1380$ index cases) reported median STI PN uptakes of: 0.9 (IOR 0.5-1.2) notified, 0.7 (IOR 0.4-0.9) evaluated, and 0.3 (IOR 0.2-0.3) new infections detected. Partner services counselling was shown to reduce reinfection rates, and no adverse events were reported. Perceived patient barriers included social stigma, fear of relationship breakdown, right to confidentiality, and lack of partner contact information. Perceived infrastructure barriers included lack of available patient education, limited resources and trained staff, mistrust between patients and health providers, and lack of partner services regulations. All studies were in heterosexual populations; none examined partner services in men who have sex with men (MSM).

Conclusions Although STI PN is not widespread in China, these limited studies suggest that more structured pilot programs could be feasible. More research on STI partner services, particularly among MSM and other high-risk groups, is an important public health priority.

\section{P5-S5.06 POPULATION-BASED PARTNER SERVICES FOR MEN WHO HAVE SEX WITH MEN (MSM) WITH GONORRHOEA OR CHLAMYDIAL INFECTION: A MISSED HIV PREVENTION OPPORTUNITY}

doi:10.1136/sextrans-2011-050108.555

${ }^{1} \mathrm{M}$ Golden, ${ }^{2} \mathrm{M}$ Stenger, ${ }^{1} \mathrm{R}$ Kerani, ${ }^{1} \mathrm{~J}$ Dombrowski, ${ }^{1} \mathrm{~J}$ Stekler. ${ }^{1}$ University of Washington, Seattle, USA; ${ }^{2}$ Washington State Department of Health, Olympia, USA

Background Few US health departments provide partner service (PS) to persons with gonorrhoea (GC) or chlamydia (CT). We sought to define the potential utility of providing PS to MSM with GC or CT as an HIV prevention intervention.

Methods We analysed public health reporting and PS data from WA State, USA, 2007-2010 to evaluate whether provider initiated case reports could identify MSM with GC or CT, to assess engagement in HIV care among MSM with STIs previously diagnosed with HIV, and to determine how often MSM HIV test when diagnosed with GC or CT. Estimates of previously diagnosed HIV, engagement with care, and HIV testing were derived from a random sample of cases weighted to reflect cases' residence and receipt of care in the King County STD clinic. We linked STI and HIV reporting data to estimate the risk of subsequent HIV diagnoses among MSM reported with GC or CT.

Results Medical providers recorded the gender of cases' sex partners on 19000 (68\%) of 27762 case reports on men with GC or CT, 4281 $(22 \%)$ of which indicated that the case had sex with men. Case report and PS interview data on cases' sexual orientation agreed in 6711 (96\%) of 6999 cases for which both data sources were available $(\kappa=0.90)$. Agreement was higher for STD clinic cases $(\kappa=0.98)$ than for other King County cases $(\kappa=0.86)$ or among men outside of King County $(\kappa=0.68)$. Among a random sample of 1103 cases identified as MSM in both case report and PS data, $25 \%$ were previously diagnosed with HIV, of whom $87 \%$ were in care and $63 \%$ were on antiretrovirals. Among MSM without a prior HIV diagnosis, 55\% reported that they HIV tested when diagnosed with GC or CT. The proportion of MSM who HIV tested was higher STD clinic cases (82\%) than among other King County MSM (57\%) or MSM outside of King County $(41 \%)(p<0.0001)$. Nineteen $(3.3 \%)$ of 583 tested MSM were newly diagnosed with HIV. Among MSM without a prior HIV diagnosis who were not diagnosed with HIV at time of their GC or CT diagnosis, the subsequent rate of new HIV diagnosis was $2.5 / 100$ person years. Among 1421 MSM diagnosed with HIV in WA State $2007-2010,6.3 \%, 9.6 \%$, and $11.9 \%$ had been reported with GC or CT in the 1, 2, and 3 years prior to HIV diagnosis, respectively.

Conclusions MSM with GC and CT are an identifiable and accessible population at high risk for HIV acquisition and transmission who might benefit from efforts to increase HIV testing frequency and linkage to HIV care. Expanding PS for bacterial STI should be evaluated as a means to achieve these goals. 